\title{
ANÁLISE GRANULOMÉTRICA DE SEDIMENTOS DE PRAIAS ARENOSAS ATRAVÉS DE IMAGENS DIGITAIS. DESCRIÇÃO DE UM PROTOCOLO DE MENSURAÇÃO DE PARTÍCULAS NO SOFTWARE IMAGEJ - FIJI.
}

\author{
GUILHERME, P.D.B. ${ }^{1,2 *}$; BORZONE, C.A. ${ }^{2}$; BUENO, M.L. ${ }^{3} \&$ LAMOUR, M.R. ${ }^{4}$ \\ 1. Programa de Pós-Graduação em Ecologia e Conservação, Universidade Federal do Paraná. Centro \\ Politécnico, Curitiba - PR, Brasil. \\ 2. Laboratório de Ecologia de Praias Arenosas, Universidade Federal do Paraná. Centro de Estudos \\ do Mar, Pontal do Paraná - PR, Brasil. \\ 3. Museu de História Natural Prof. Adão José Cardoso, Universidade Estadual de Campinas. Instituto \\ de Biologia, Campinas - SP, Brasil. \\ 4. Laboratório de Oceanografia Geológica, Universidade Federal do Paraná. Centro de Estudos do \\ Mar, Pontal do Paraná - PR, Brasil. \\ *Corresponding author: pdbguilherme@gmail.com
}

\begin{abstract}
Guilherme, P.D.B.; Borzone, C.A.; Bueno, M.L. \& Lamour, M.R., 2015. Análise granulométrica de sedimentos de praias arenosas através de imagens digitais. Descrição de um protocolo de mensuração de partículas no software ImageJ - Fiji. Braz. J. Aquat. Sci. Technol. 19(2). eISSN 1983-9057. DOI: 10.14210/bjast.v19n2. The particle size analysis of sandy sediments can be performed using different techniques such as sieving, decantation, laser diffraction or digital image analysis. The digital image analysis may be considered a fast and lower cost technic, and can be practice in any place with a scanner and computer. However, most studies describe that method use complex and commercial software. The aim of this study is to describe and evaluate a methodological protocol of easy access and low cost for digital image analysis using the software ImageJ. Results of ten samples of sandy sediments using the described protocol were compared with the results of particle size analysis done by sieving and laser diffraction techniques. In general, image analysis and laser diffraction had similar results. These results agreed in $60 \%$ of the textual classifications (considering the statistical parameters of mean and selection). In dissimilar classifications, the absolute values were very close to the limits of those classifications, with differences of only $0.1 \varnothing$. Thus, digital image analysis using free software ImageJ represents a practical, low cost and easy accessibility technic for granulometric analysis.
\end{abstract}

Keywords: Image analysis, Particle size, Sediment, Beach, ImageJ - Fiji.

\section{INTRODUÇÃO}

A análise granulométrica nos permite estabelecer, de forma quantitativa, a distribuição textural das partículas sedimentares (Suguio, 1973). Desta forma é possível caracterizar, classificar e correlacionar sedimentos de locais diferentes por meio de tratamento estatístico.

Por várias décadas, a análise granulométrica mais utilizada foi o peneiramento e posterior pesagem das frações de uma amostra sedimentológica (Krumbein \& Pettijohn, 1938; Suguio, 1973). Outras técnicas incluem a decantação dos grãos de sedimento através de uma coluna d'água (Gibbs, 1972; Syvitski et al., 1991), a difração de laser (Agrawal et al., 1991), que apresenta resultados rápidos e precisos, mas que possuem um alto custo financeiro pela aquisição e manutenção do equipamento, e a mais recente técnica que utiliza imagens digitais para medir as partículas dos sedimentos (Fortey, 1995; Francus, 1998).

Atualmente, a análise granulométrica feita por difração de laser é a mais utilizada devido à facilidade, confiabilidade no procedimento metodológico (Storti
\& Balsamo, 2010), e por proporcionar a automação e uniformidade dos resultados (Eshel et al., 2004; Stojanović et al., 2012). Contudo, muitos autores (Eshel et al., 2004; Kelly \& Etzler, 2014) criticam a técnica e listam falsas premissas sobre as quais o método está apoiado, e.g. a orientação randômica das partículas e a determinação das frequências com base em resultados de diâmetro esférico equivalente. Progressivamente, estas dificuldades estão sendo superadas por equipamentos mais modernos, que utilizam mais que um feixe de luz na tentativa de analisar de forma complementar o mesmo conjunto de partículas sedimentares submetido à análise.

A análise de imagens é um campo em próspero crescimento devido aos diversos usos dessa ferramenta (Schneider et al., 2012). A sequência típica de operações para a análise de imagens digitais inclui a aquisição e processamento da imagem, a mensuração das partículas e o processamento e interpretação dos dados (Bons \& Jessel, 1996). Através desse método pode-se estudar, além das variáveis métricas (ex.: diâmetro dos grãos), algumas características adicionais como formato, composição do grão (Lira \& Pina, 
2011), e posição dos grãos em um testemunho (Rubin, 2004). Essa técnica pode ser aplicada através de duas rotinas distintas: analisando grãos previamente coletados (Francus, 1998; Lira \& Pina, 2009; 2011) ou através da fotografia feita em campo (Rubin, 2004).

Pina \& Lira (2009) publicaram uma comparação entre as alternativas acima descritas a fim de validar a utilização dessa técnica. Germán-Rodriguez \& Uriarte (2009) chegaram à conclusão de que a difração de laser apresenta resultados diferentes do peneiramento, destacando ainda que as técnicas que utilizam imagem digital são rápidas e precisas na determinação das distribuições de tamanho dos grãos de sedimentos, tendo resultados similares ao peneiramento.

A caracterização dos sedimentos através da determinação da distribuição de tamanho dos seus grãos é de vital importância para estudos, tanto de cunho geológico quanto biológico, de um ambiente praial (Hardisty, 1990; McLachlan, 1990). Por isso, a grande maioria dos trabalhos de ecologia de praias utiliza esta caracterização como uma das principais variáveis abióticas que controlam as comunidades biológicas (Defeo et al., 1992; Borzone et al., 1996; Alves \& Pezzuto, 2009).

O objetivo do presente trabalho é apresentar um protocolo metodológico baseado nos artigos de Lira \& Pina (2009; 2011), que utilizam a análise da imagem para caracterização granulométrica de sedimentos através do pacote Fiji do software Image J, considerando as alterações sugeridas por Francus \& Pirard (2004), que esclarecem os principais erros cometidos nas análises de imagem de partículas de sedimentos. Os resultados desse protocolo foram comparados com os resultados de análises granulométricas feitas nas mesmas amostras através do método de peneiramento e de difração de laser.

\section{DESCRIÇÃO DO MÉTODO}

\section{Software}

O Image é um programa livre de análise de imagens com código aberto, amplamente utilizado, que funciona em múltiplas plataformas (Rasband, 2013; Schneider et al., 2012). Por isso existem diversos pacotes com diferentes ferramentas aplicadas às distintas áreas do conhecimento. Dentre esses pacotes, o Fiji é muito utilizado nas ciências naturais. Este pacote de processamento de imagem complementa o software propriamente dito com: o Java, o Java 3D e diversos outros plug-ins organizados em uma estrutura coerente. O Fiji garante uma fácil instalação e função de atualização automática, além de uma documentação completa no próprio site do desenvolvedor (Schindelin et al., 2012).

\section{Aquisição de imagem}

A rotina de aquisição de imagens consiste na deposição dispersa de sedimentos sobre a bandeja de vidro transparente de um escâner, tentando sempre minimizar a sobreposição das mesmas. Desta forma, considera-se que as partículas de sedimentos tendem a cair com o seu eixo maior e intermediário paralelo ao plano do vidro do escâner.

Para facilitar a posterior retirada dos sedimentos do escâner, pode-se colocar as partículas sobre um vidro, papel de transparência ou cobrir o escâner com papel filme (PVC), cuidando para que não haja riscos ou bolhas que ocasionem ruídos na análise.

Dependendo das cores dos sedimentos, um fundo branco ou preto pode ser colocado sobre os sedimentos a fim de evitar a presença de sombras. Uma escala gráfica para posterior mensuração na imagem adquirida é necessária (Ex. régua, escala adquirida em lojas especializadas de fotografia ou ainda pode-se imprimir uma escala no site http://web. ncf.ca/jim/scale/).

A imagem dos grãos de sedimentos é escaneada com uma resolução espacial mínima de 1200dpi (dots per inch) ou aproximadamente $0,021 \mathrm{~mm}$ por pixel (Fig. 1). Esta imagem pode ser recortada em imagens menores para ter posteriormente seus resultados somados, ou analisadas por inteiro, o que requer um computador com alta memória RAM (>2GB). A imagem pode ser adquirida colorida ou em escala de cinzas, e então salva na extensão “*.tiff”. Desta forma, as menores estruturas na imagem devem corresponder a sedimentos de 3 por 3 pixels (i.e. $0,0625 \mathrm{~mm}$ ou $4 \varnothing$ ), correspondendo ao limite inferior das areias muito finas na escala de Wentworth (1922).

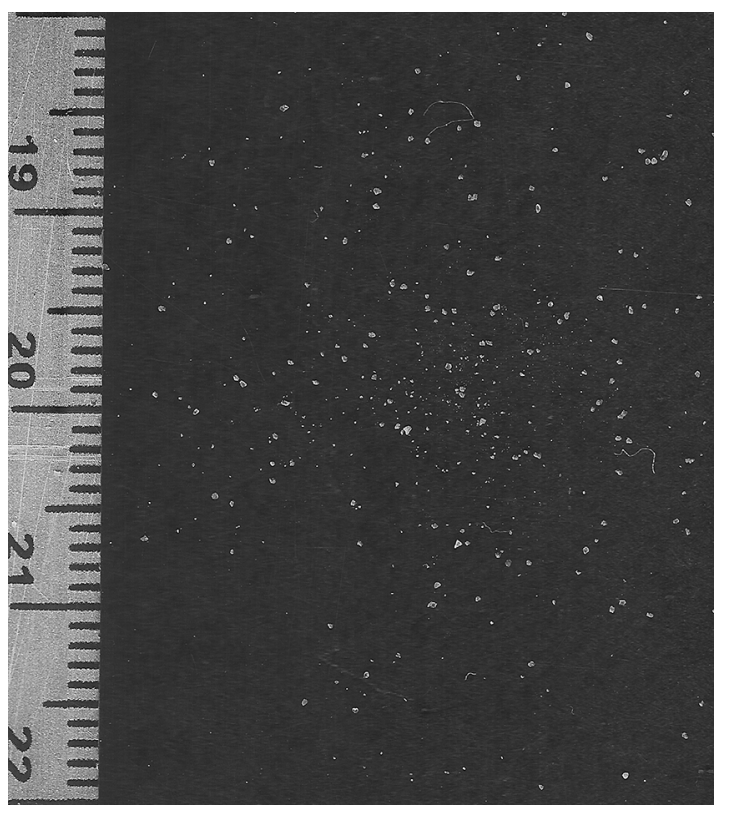

Figura 1 - Imagem adquirida pelo escâner das partículas de sedimentos (pontos brancos) em fundo preto junto com a escala (régua). 


\section{Tratamento e preparação da imagem}

Para o tratamento e preparação da imagem, seguem-se os seguintes passos detalhados a seguir, conforme sumarizado na Figura 2.

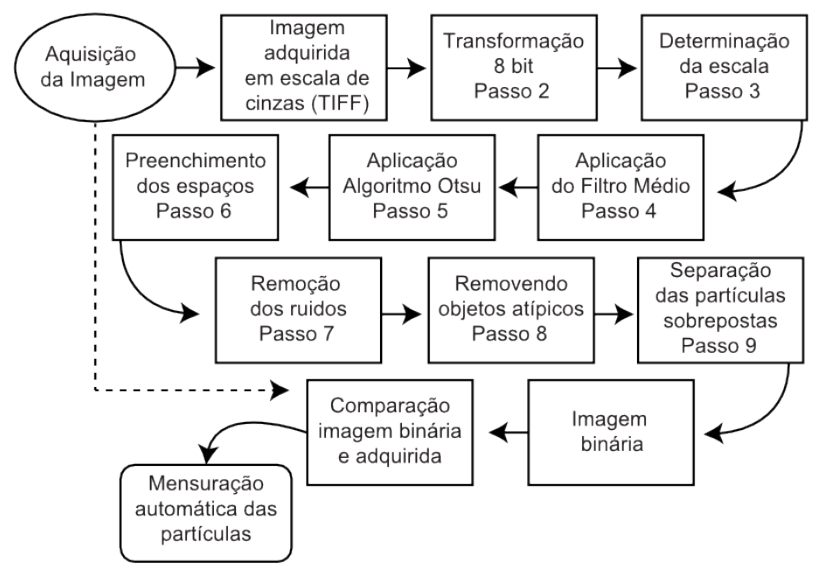

Figura 2 - Diagrama da sequência de passos para o processamento das imagens.

O aspecto geral do software ImageJ - Fiji, mostra uma pequena tela com menus e diversas ferramentas (Fig. 3).

Passo 1 - Abre-se o arquivo a ser analisado, menu: FILE - OPEN ou arrastando a figura que se deseja abrir para o programa.

Passo 2 - Transforma-se a imagem em 8-bit, menu: IMAGE - TYPE - 8-BIT.

III (Fiji Is Just) ImageJ
File Edit Image Process Analyze Plugins Window Help
$\mid \square$

Figura 3 - Janela principal do software ImageJ - Fiji.

Passo 3 - Ajusta-se a escala através da ferramenta linha (straight) no quinto ícone de ferramentas, traçando uma reta sobre a escala conhecida. No menu: ANALYSE - SET SCALE, na janela a seguir (Fig. 4) é digitada a distância real medida (Know distance) com a sua respectiva unidade (Unit of length: $\mathrm{mm}$, $\mathrm{cm}, \mathrm{m}, \mathrm{km}$ ).

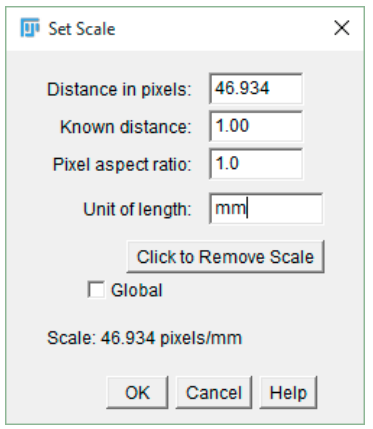

Figura 4 - Janela "Set Scale" do software ImageJ - Fiji.
A partir de agora, qualquer mensuração realizada pelo software será feita na escala determinada. Através da ferramenta de seleção retangular é possível selecionar a área de interesse que terá os grãos mensurados, podendo-se até cortar a figura (Menu: IMAGE - CROP) mantendo a configuração da escala, mas sem a imagem da régua (Fig. 5).

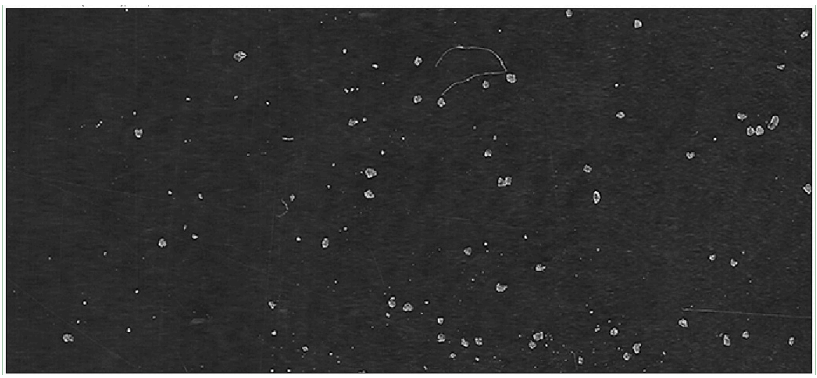

Figura 5 - Imagem ampliada das partículas de sedimentos (em branco) com fundo preto e sem o ruído criado pela presença da régua (escala).

Passo 4 - Para reduzir o ruído da imagem causada pela iluminação desigual (Starkey \& Samantaray, 1991; 1994) aplica-se um filtro de convolução espacial média, no menu: PROCESS FILTERS - MEAN. Este filtro, quando aplicado, deixa a imagem desfocada reduzindo o ruído produzido pela iluminação e pelo fundo. Utilizando valores menores de 3 pixels de raio, ele desfoca qualquer imagem causada pela iluminação, podendo-se escolher o valor mais adequado pela opção "preview".

Passo 5 - Para segmentar a imagem (ou seja, separar as partes de interesse do fundo) é necessário ter um valor limiar de níveis de cinza após uma análise dos histogramas dos mesmos. Este limiar criará a imagem binária onde os objetos (partículas de sedimentos) são representados por pixels pretos ou brancos. Sugere-se a utilização do algoritmo proposto por Otsu (1979), que usa uma técnica não paramétrica e não supervisionada para a seleção automática do limiar, no menu: IMAGE - ADJUST - THRESHOLD, já que o mesmo é amplamente utilizado (Lira \& Pina, 2011). Após a seleção do algoritmo automático, deve-se desmarcar a opção de fundo preto (Dark background) antes de aplicá-lo (Apply), produzindo uma imagem semelhante à Figura 6.

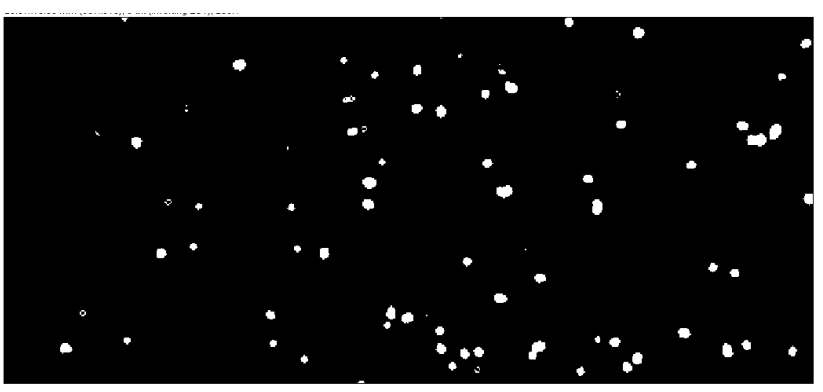

Figura 6 - Imagem depois de segmentada e sem as aberturas. 
Passo 6 - Caso apareçam imagens de grãos com aberturas ou espaços internos (devido a incidência da luz do escâner sobre sedimentos translúcidos que possuem estes espaços devido a inclusões fluídas originárias do processo de solidificação do cristal), deve-se preencher os mesmos através do procedimento PROCESS - BINARY - FILL HOLES.

Passo 7 - Pequenos objetos indesejados e ruídos da imagem (Outliers) podem ser removidos através do menu PROCESS - NOISE - REMOVE OUTLIERS (Abramoff et al., 2004; Russ, 1990). Na janela seguinte (Fig. 7), o raio (Radius) e o limiar (Threshold) são determinados bem como a aparência desses objetos atípicos. Nesta análise, foram usados os valores de 3 pixels de raio e 30 de limiar.

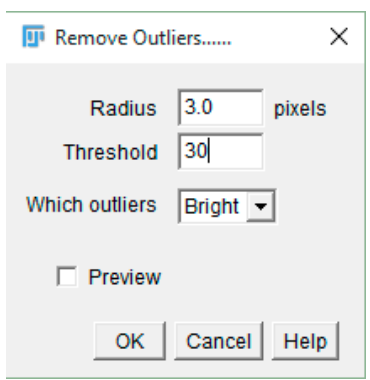

Figura 7 - Janela de opções para a retirada de outliers da imagem segmentada.

Passo 8 - Mesmo depois dos diversos tratamentos aplicados à imagem para a retirada do ruído, algumas anomalias ainda podem permanecer, como por exemplo, imperfeições aparentes no vidro do escâner ou ainda bolhas ou riscos causados quando utilizado um papel filme para proteger o escâner (Fig. 8). A melhor maneira de retirar esses objetos é manualmente com a ferramenta Pincel (Brush) na cor do fundo, ou tentando evitar ao máximo que eles sejam incluídos ao se recortar a imagem no passo 3 .

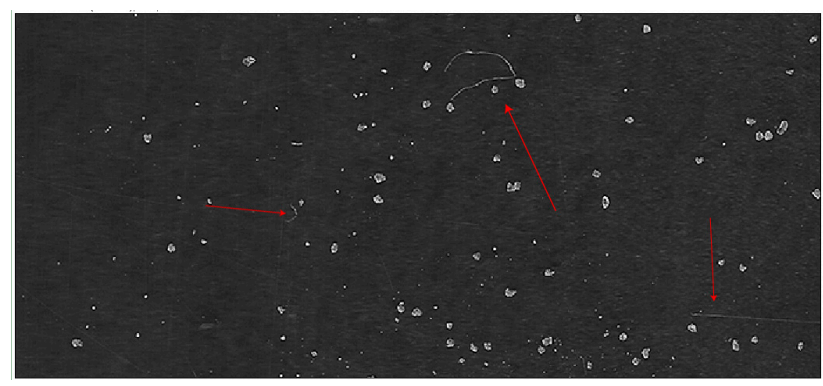

Figura 8 - Exemplo de anomalias indicadas pelas setas.

Passo 9 - Mesmo sendo cuidadoso na hora de depositar os sedimentos na superfície do escâner, algumas partículas podem ficar sobrepostas. Para separá-las de forma automática, pode-se utilizar o menu: PROCESS - BINARY - WATERSHED (Fig. 9).

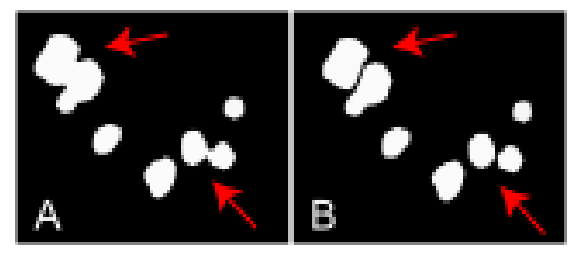

Figura 9 - Objetos sobrepostos (A) e posteriormente os objetos separados com a aplicação do Watershed (B).

No final de todos os processos acima descritos, a imagem binária pode ser comparada com a imagem original em escala de cinza (lado a lado) para confirmar se os objetos foram segmentados corretamente e se não houveram distorções durante o processamento da imagem (Bouabid et al., 1992) (Fig. 10).

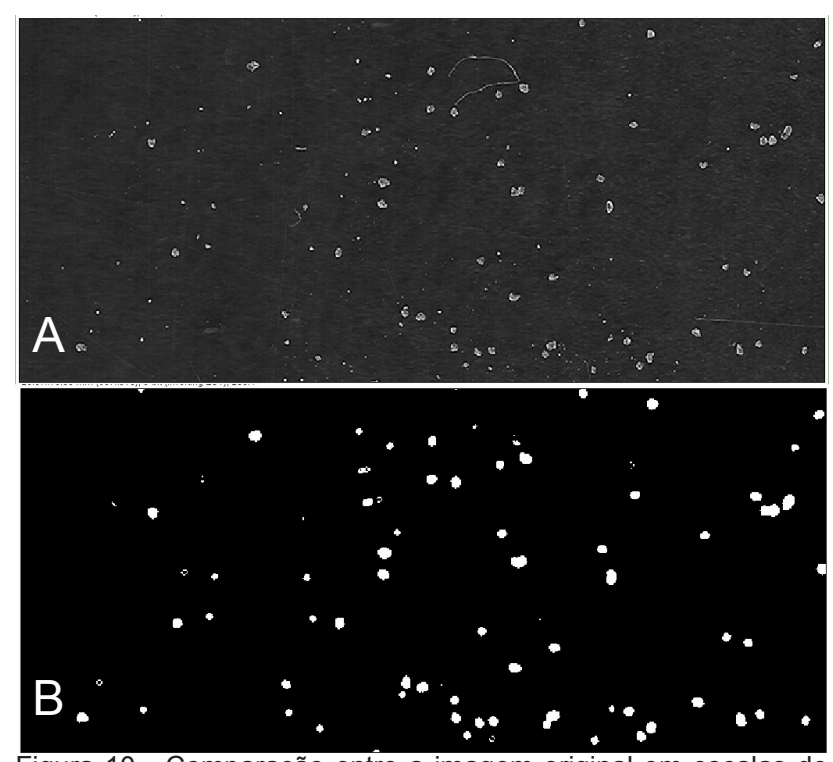

Figura 10 - Comparação entre a imagem original em escalas de cinza (8-bit) adquirida pelo escâner (A) e a imagem binarizada (B).

\section{Mensuração das Partículas}

Através do menu: ANALYZE - SET MEASUREMENTS é possível configurar as opções que o software irá utilizar durante o processo de análise das partículas. Para as análises granulométricas deve-se selecionar as opções: área (Area) que calcula a área de cada partícula e ajuste de elipse (Fit elipse) que cria uma elipse sobre a partícula e calcula o eixo maior e o menor e o ângulo entre eles. Outras opções interessantes em análises mineralógicas e morfológicas são o centro de massa (Center of Mass) que determina a posição $\mathrm{X}$ e $\mathrm{Y}$ de cada partícula, os descritores de forma (Shape descriptors) que medem a circularidade, a razão entre os eixos, a esfericidade e a solidez das partículas, e finalmente o perímetro (Perimeter) que mede o comprimento do limite exterior. Além disso, na opção casas decimais (Decimal places) é possível determinar o número de decimais que os resultados terão. 
Para que o programa analise as partículas automaticamente, no menu "ANALYZE" acesse "ANALYZE PARTICLES" (Fig. 11). O primeiro campo a ser preenchido é o tamanho (Size), que neste caso é expresso em $\mathrm{mm}^{2}$ e deve englobar o tamanho máximo e mínimo das partículas a serem mensuradas. Por exemplo, na análise de partículas de sedimentos de praias arenosas, o diâmetro dos grãos pode variar de $0,0625 \mathrm{~mm}$ (limite inferior da areia muito fina) até no máximo dois milímetros (limite inferior dos cascaIhos). Então completa-se o campo com: "0.0039-4". O segundo campo a ser completado é a circularidade (Circularity), que varia de zero (nada esférico) até um (completamente esférico).

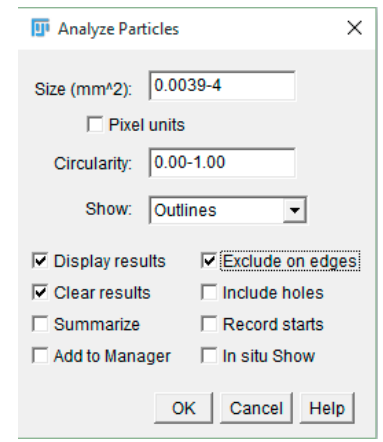

Figura 11 - Janela Analyze Particles do software ImageJ - Fiji.

O terceiro campo é uma série de opções que vai determinar como os objetos medidos serão mostrados. Quando selecionado Outlines uma janela mostrará as partículas analisadas através de esboços numerados, enquanto Masks mostrará os contornos cheios e Ellipses mostrará apenas as elipses sobrepostas às partículas (Fig. 12)

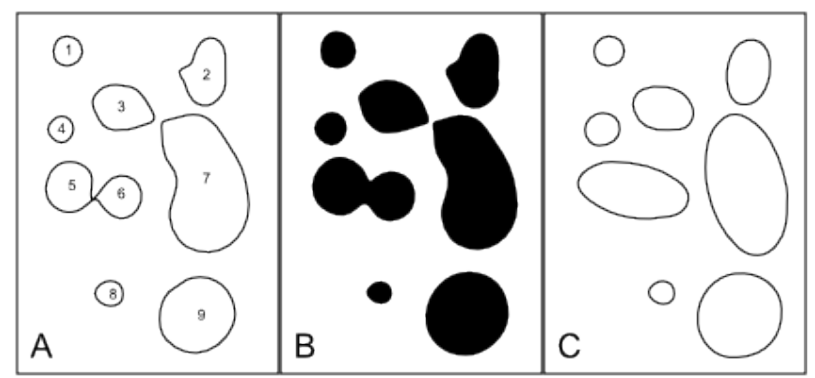

Figura 12 - Resultados do analisador de partículas do ImageJ (Modificado de http://rsbweb.nih.gov/ij/).

Outras opções que podem ainda ser selecionadas são: mostrar resultados (Display Results), que exibe uma nova janela com as medições para cada partícula; apagar resultados (Clear Results), para apagar quaisquer resultados das medições anteriores; resumir (Summarize), para exibir, em uma nova janela, a contagem de partículas, a área total da partícula, o tamanho médio de partícula e a fração de área; excluir nas bordas (Exclude on Edges), para ignorar as partículas que estão tocando a borda da imagem ou seleção (sugerido por Francus \& Pirard, 2004).

Após a análise das partículas, o software disponibiliza, através de uma janela independente, as partículas que foram consideradas (Fig. 13) e os dados em forma de tabela, que podem ser salvos em planilhas do Excel ( ${ }^{*}$. xls) ou em texto ( ${ }^{*}$.txt) (Fig. 14).

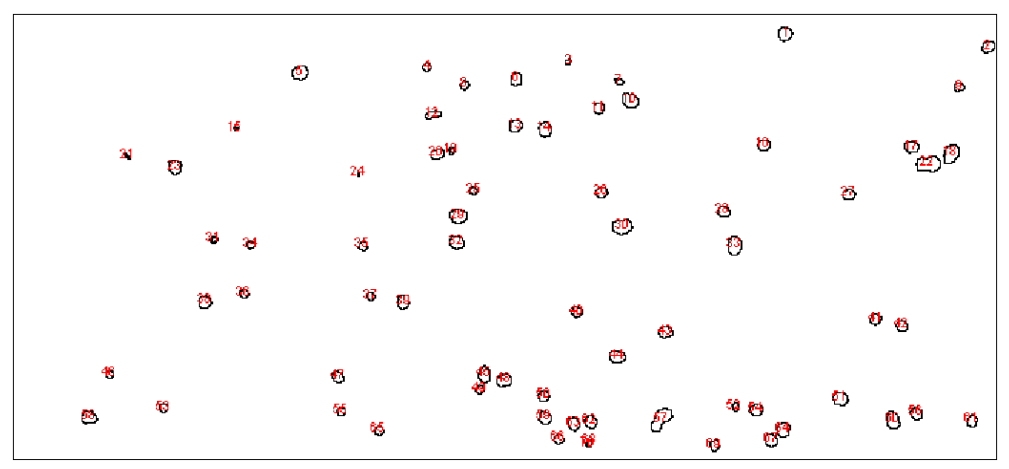

Figura 13 - Partículas mensuradas automaticamente pelo software ImageJ - Fiji no modo Outlines.

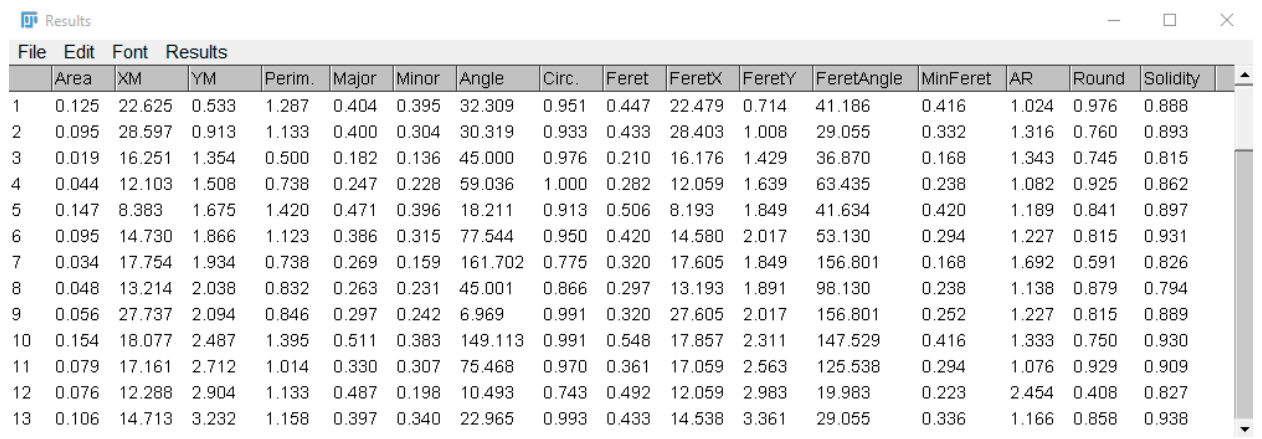

Figura 14 - Janela contendo as informações mensuradas pelo software Image - Fiji. 


\section{Tratamento dos dados}

Afim de tornar os dados adquiridos pela análise de imagem comparáveis com os demais métodos se faz necessária a seleção ou transformação das variáveis mensuradas. Para a comparação com o método de peneiramento, o eixo que controla as passagens das partículas através das aberturas de peneira é o eixo intermediário (Sahu, 1965; Fritz \& Moore, 1988). $\mathrm{Na}$ análise de imagem esse eixo consiste no diâmetro menor (Minor na planilha, Fig. 14) obtido pelo software. Contudo, alguns autores sugerem a utilização de outra variável, como Francus (1998) que recomenda o uso de um diâmetro equivalente $\left(D_{0}\right)$, também utilizado nos analisadores de difração de laser, que pode ser calculado pela área do grão (Equação 1).

$$
D_{0}=2 \sqrt{\frac{\text { Área }}{\pi}} \quad \text { Equação } 1
$$

Desta forma, podemos utilizar o diâmetro menor ou o equivalente para os futuros cálculos dos parâmetros estatísticos da análise granulométrica (média, moda, desvio padrão, curtose e assimetria). Lembrando que os dados obtidos são adquiridos em milímetros, devendo se fazer a conversão para a escala $\varnothing$ (phi $=-\log _{2}$ diâmetro mm) (Krumbein, 1934) caso seja necessário.

\section{Teste dos Métodos}

Para o teste dos métodos foram utilizadas dez amostras provenientes da sedimentoteca do Laboratório de Oceanografia Geológica do Centro de Estudos do Mar (CEM/UFPR). Tais amostras foram coletadas na zona de espraiamento de diferentes praias do litoral do Paraná, para um estudo relativo à identificação de células de deriva longitudinal litorânea realizado por Alves (2011).

Todas as amostras foram processadas pelas três técnicas de análise granulométrica (peneiramento, difração de laser e análise de imagem seguindo o presente protocolo e utilizando o diâmetro menor como medida do grão). Além disso, as amostras foram submetidas às análises contendo os carbonatos, ou seja, sem ataque químico para a sua eliminação. Para que a comparação entre os métodos fosse uniforme foram computadas as frequências de distribuições de tamanho dos grãos com classes de $0,5 \varnothing$ (padrão do peneiramento).

O peneiramento foi realizado segundo as premissas descritas por Suguio (1973). Após homogeneização e quarteamento manual da amostra, os sedimentos foram levados à estufa $\left(60^{\circ} \mathrm{C}\right)$ para secagem durante 24 horas. Uma fração desses sedimentos, com aproximadamente $100 \mathrm{~g}$, foi destinada à elutriação. $O$ método consiste em submeter os sedimentos à pas- sagem de um fluxo ascendente contínuo de água, o qual tem potencial de suspensão para as partículas finas $(<0,062 \mathrm{~mm})$. Os sedimentos elutriados foram acondicionados em papel filtro semi-qualitativo e novamente levados à estufa para secagem. Posteriormente foram submetidos à agitação mecânica durante um período de 10 minutos em um conjunto de 12 peneiras, com malhas entre $4,0 \varnothing(0,062 \mathrm{~mm})$ e $-1,5 \varnothing(4$ $\mathrm{mm}$ ) em intervalos de $0,5 \varnothing$, de acordo com a escala granulométrica proposta por Wentworth (1922). As frações encontradas foram então pesadas em balança analítica de precisão $(0,001 \mathrm{~g})$ de forma a determinar as frequências granulométricas.

Cerca de $1 \mathrm{~g}$ de amostra passou por um analisador de tamanho de partículas por difração de laser (granulomêtro), modelo Microtrac S3500 BlueWave. A determinação do tamanho dos grãos neste equipamento é baseada na interação entre três feixes de laser vermelho $(\lambda=780 \mathrm{~nm})$ e a partícula de sedimento. Além disso, o software Microtrac FLEX, utiliza a teoria de compensação de Mie, que é mais recomendada (ISO 13320-1, 2009) para a determinação do volume em cada uma das classes. Os resultados foram configurados através do software do dispositivo para que as frações fossem determinadas nas mesmas classes de frequência que no peneiramento.

As tabelas de frequência de classes de tamanho dos três métodos foram analisadas no software GRADISTAT (Blott \& Pye, 2001) para a determinação dos parâmetros estatísticos granulométricos (diâmetro médio e grau de seleção). Os resultados foram expressos em $\varnothing$ e calculados pelo método de Folk \& Ward (1957). A média e grau de seleção foram testados estatisticamente através de Análise de Variância (ANOVA) de medidas repetidas utilizando os métodos como fatores, as diferenças foram evidenciadas a posteriori através de um teste Student-Newman-Keuls (SNK).

\section{RESULTADOS}

De forma geral, as três técnicas apresentam resultados semelhantes (Tabela 1) com classificações textuais iguais em $60 \%$ dos casos (onde coincidiram tanto na média quanto no grau de seleção). Quando considerada apenas a média, os três métodos concordaram em $90 \%$ dos casos, onde apenas a amostra \#21 foi classificada como areia média pela difração de laser, enquanto as demais técnicas a classificaram como areia fina. Quando considerado o grau de seleção, as técnicas concordam em $70 \%$, com duas classificações (\#30 e \#34) díspares na difração de laser (bem selecionado ao invés de moderadamente selecionado) e uma classificação (\#45) na análise de imagem (moderadamente selecionado ao invés de 
bem selecionado).

Através da análise de imagem, podemos ter outros resultados, como os descritos na tabela 2 que apresentam valores médios para o perímetro, eixo maior e menor, além da circularidade e esfericidade das partículas.

Na comparação quantitativa, a ANOVA demonstrou diferença entre os métodos, tanto na mensuração da média $\left(F_{2,18}=20,93 ; p<0,05\right)$ quanto na seleção
$\left(F_{2,18}=6,78, p<0,05\right)$. Apesar disso, quando analisados os testes a posteriori as diferenças nas médias estiveram atreladas ao peneiramento, já que não foram evidenciadas diferenças entre a difração de laser e a análise de imagem (Tabela 1). Para o grau de seleção, as diferenças no teste a posteriori formaram dois agrupamentos, com a análise de imagem em um lado e a difração em outro, e entre as duas o peneiramento.

Tabela 1 - Comparação entre a média e graus de seleção de diferentes métodos de análise granulométrica obtidos através das tabelas de frequência das classes de tamanho das partículas $(0,5 \varnothing)$ e calculados pelo software GRADISTAT. AM: Areia Média; AF: Areia Fina; MS: Moderadamente selecionada; BS: Bem selecionada.

\begin{tabular}{cccc|ccc|ccc} 
& \multicolumn{3}{c|}{ Análise de Imagem } & \multicolumn{3}{c|}{ Difração de Laser } & \multicolumn{3}{c}{ Peneiramento } \\
Amostra & Média & Seleção & Class. & Média & Seleção & Class. & Média & Seleção & Class. \\
\hline$\# 01$ & 1,828 & 0,6752 & AM/MS & 1,741 & 0,7158 & AM/MS & 1,863 & 0,6570 & AM/MS \\
$\# 11$ & 2,059 & 0,5857 & AF/MS & 2,006 & 0,5066 & AF/MS & 2,314 & 0,6080 & AF/MS \\
$\# 21$ & 2,116 & 0,6996 & AF/MS & 1,960 & 0,5193 & AM/MS & 2,286 & 0,5340 & AF/MS \\
$\# 24$ & 2,402 & 0,6041 & AF/MS & 2,283 & 0,5254 & AF/MS & 2,747 & 0,7270 & AF/MS \\
$\# 28$ & 2,465 & 0,6737 & AF/MS & 2,243 & 0,5263 & AF/MS & 2,526 & 0,6660 & AF/MS \\
$\# 30$ & 2,225 & 0,6220 & AF/MS & 2,419 & 0,4906 & AF/BS & 2,606 & 0,5810 & AF/MS \\
$\# 33$ & 2,386 & 0,6109 & AF/MS & 2,485 & 0,5443 & AF/MS & 2,711 & 0,5130 & AF/MS \\
$\# 34$ & 2,260 & 0,5554 & AF/MS & 2,373 & 0,4778 & AF/BS & 2,749 & 0,5370 & AF/MS \\
$\# 45$ & 2,415 & 0,5522 & AF/MS & 2,460 & 0,3901 & AF/BS & 2,77 & 0,3700 & AF/BS \\
$\# 47$ & 2,146 & 0,4865 & AF/BS & 2,527 & 0,4318 & AF/BS & 2,945 & 0,4120 & AF/BS \\
\hline SNK & a & a & & $a$ & b & & $b$ & Ab &
\end{tabular}

Tabela 2 - Valores médios (desvio-padrão) do perímetro, eixo maior e intermediário, circularidade e esfericidade das partículas de sedimento obtidos através de análise de imagem em cada uma das amostras processadas.

\begin{tabular}{|c|c|c|c|c|c|c|c|c|c|c|c|}
\hline \multirow[t]{2}{*}{ Amostra } & \multirow[t]{2}{*}{$n$} & \multicolumn{2}{|c|}{$\begin{array}{c}\text { Perímetro } \\
(\mathrm{mm})\end{array}$} & \multicolumn{2}{|c|}{$\begin{array}{l}\text { Eixo } \\
\text { Maior } \\
(\mathrm{mm})\end{array}$} & \multicolumn{2}{|c|}{$\begin{array}{c}\text { Eixo } \\
\text { Intermediário } \\
(\mathrm{mm})\end{array}$} & \multicolumn{2}{|c|}{$\begin{array}{c}\text { Circularidade } \\
\frac{4 \pi * \text { área }}{\text { perímetro }^{2}}\end{array}$} & \multicolumn{2}{|c|}{$\begin{array}{l}\text { Esfericidade } \\
\text { eixo maior } \\
\text { eixo interm. }\end{array}$} \\
\hline & & $\bar{x}$ & $\sigma$ & $\bar{x}$ & $\sigma$ & $\bar{x}$ & $\sigma$ & $\bar{x}$ & $\sigma$ & $\bar{x}$ & $\sigma$ \\
\hline$\# 01$ & 9.869 & 0,8916 & 0,4384 & 0,3086 & 0,1447 & 0,2165 & 0,1009 & 0,8500 & 0,1083 & 0,7160 & 0,1350 \\
\hline \#11 & 13.144 & 0,7328 & 0,2944 & 0,2579 & 0,1028 & 0,1813 & 0,0683 & 0,8696 & 0,1026 & 0,7212 & 0,1358 \\
\hline$\# 21$ & 10.552 & 0,7348 & 0,3411 & 0,2583 & 0,1184 & 0,1802 & 0,0795 & 0,8639 & 0,1206 & 0,7175 & 0,1483 \\
\hline$\# 24$ & 8.596 & 0,5890 & 0,2379 & 0,2106 & 0,0816 & 0,1420 & 0,0543 & 0,8651 & 0,1152 & 0,6929 & 0,1430 \\
\hline$\# 28$ & 12.855 & 0,5838 & 0,2758 & 0,2071 & 0,0921 & 0,1410 & 0,0618 & 0,8667 & 0,1191 & 0,7019 & 0,1418 \\
\hline$\# 30$ & 17.631 & 0,6602 & 0,2945 & 0,2337 & 0,1037 & 0,1625 & 0,0654 & 0,8744 & 0,1166 & 0,7204 & 0,1455 \\
\hline \#33 & 16.415 & 0,6028 & 0,2639 & 0,2140 & 0,0875 & 0,1446 & 0,0592 & 0,8615 & 0,1203 & 0,6938 & 0,1442 \\
\hline$\# 34$ & 12.386 & 0,6123 & 0,2344 & 0,2171 & 0,0852 & 0,1553 & 0,0538 & 0,8944 & 0,1076 & 0,7377 & 0,1377 \\
\hline$\# 45$ & 14.928 & 0,5709 & 0,2006 & 0,2044 & 0,0705 & 0,1394 & 0,0459 & 0,8723 & 0,1054 & 0,6998 & 0,1384 \\
\hline \#47 & 18.664 & 0,6564 & 0,2255 & 0,2327 & 0,0821 & 0,1637 & 0,0493 & 0,8815 & 0,1047 & 0,7251 & 0,1370 \\
\hline
\end{tabular}

Apesar dos resultados das classificações textuais serem diferentes entre os métodos, foi possível observar que existe uma proximidade numérica entre os resultados. Por exemplo, na amostra \#21, o valor de média calculado com a análise de imagem é de $2,116 \varnothing$ (areia fina) enquanto que na difração de laser o valor é de $1,960 \varnothing$ (areia média), sendo ambos os valores definidos como os limites dessas classificações. Na figura 15 é possível observar detalhadamente as sutis diferenças entre a análise de imagem e a difração de laser. Na maioria das amostras a difração de laser apresenta resultados superestimados em relação à análise de imagem, nos demais casos (e.g. \#33 e \#45) esse padrão parece se alternar entre as análises. 

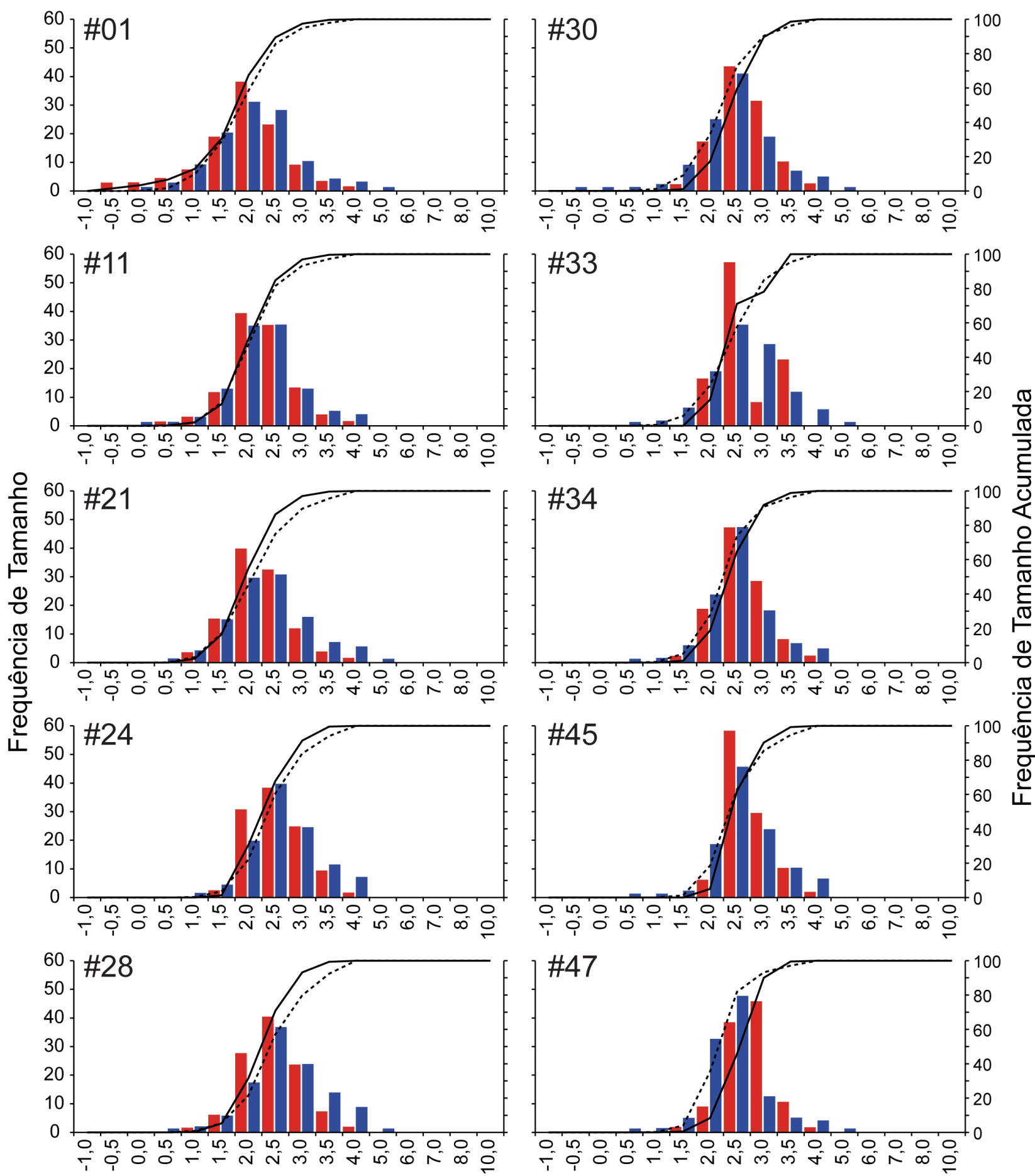

\section{Classes de Tamanho $(\varnothing)$

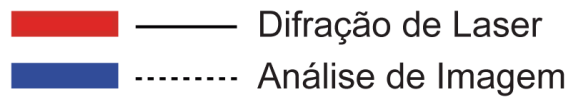

Figura 15 - Distribuições de tamanho de grão $(\varnothing)$ avaliados através do método de análise de imagem digital (barras azuis e linha pontiIhada) e difração de laser (barras vermelhas e linha contínua). O eixo horizontal representa as classes de tamanho e no eixo vertical as frequências (habitual ao lado esquerdo e acumulada no direito). 


\section{DISCUSSÃO}

Os resultados obtidos a partir das técnicas de análise de imagem e de difração de laser foram mais semelhantes entre si que aqueles obtidos pelo peneiramento. Francus (1998) evidencia que o método de análise de imagem apresenta dados de alta resolução, com ótima precisão e muita rapidez na aquisição dos resultados, contudo o método é suscetível a erros que podem ser minimizados, desde que estes erros sejam previamente identificados (Francus \& Picard, 2004). Pina \& Lira (2004) consideram ainda o método rápido e robusto, com curvas acumulativas que expressam estimativas realistas das distribuições de tamanho de grão.

Nossos resultados mostram um padrão similar aos resultados da comparação entre métodos feita por Pina \& Lira (2009), onde a difração de laser superestima os tamanhos de partícula em relação as outras duas técnicas. Germán-Rodriguez \& Uriarte (2009) evidenciam que o resultado da análise por difração de laser é influenciado pelo formato das partículas. Isso por que esse método mede o tamanho das áreas de secção transversal dos grãos, de acordo com o ângulo da luz difratada.

A análise granulométrica feita por peneiramento é muitas vezes utilizada pelo baixo custo na aquisição do equipamento e no processamento das amostras, mas depende de treinamento técnico especializado para não ser suscetível a erros humanos.

A difração de laser é um método rápido e descomplicado, contudo possuiu um alto valor na aquisição e manutenção do equipamento, com a necessidade de um técnico previamente treinado e especializado a disposição. Seus resultados são questionados por muitos autores, mas quando comparado ao peneiramento é menos susceptível a erros metodológicos. Nos casos em que análises de sedimento são feitas de forma regular e com um grande volume de amostras, esse tipo de equipamento é realmente necessário.

A análise de imagem utiliza equipamentos simples e que muitas vezes já estão disponíveis num laboratório ou gabinete de trabalho, mas também demanda de certo treinamento e um alto dispêndio de tempo. Ainda assim, é uma alternativa viável justamente por ter um custo operacional baixo e apresentar resultados fiéis que podem ser gerados pelo próprio pesquisador.

Neste contexto, a utilização de softwares livres e de simples utilização é um forte condicionante para o sucesso desta análise através de imagens digitais. Desta forma, a análise de imagem digital utilizando o programa livre ImageJ surge como uma alternativa prática, de baixo custo e de fácil acesso para a maioria dos pesquisadores, especialmente quando as análises granulométricas são feitas de forma ocasional e com baixo número de amostras.

\section{AGRADECIMENTOS}

Os autores agradecem a Dr. Leonardo Cruz da Rosa por testar o protocolo e fazer sugestões e a CAPES e a PRPPG/UFPR que possibilitaram a compra do granulômetro pelo Edital Pró-equipamentos 2011. P.D.B. Guilherme agradece a CAPES pela bolsa de doutorado.

\section{REFERÊNCIAS}

Abramoff, M.D.; Magalhães, P.J. \& Ram, S.J. 2004. Image Processing with ImageJ. Biophotonics International, 11(7):36-42.

Agrawal, Y.C.; Mccave, I.N. \& Riley, J.B. 1991. Laser diffraction size analysis. In: SYVITSKI J.M.P. (ed) Principles, Methods and Application of Particle Size Analysis. Cambridge University Press, Cambridge, 119-128p.

Alves, L.C. 2011. Identificação de células de deriva longitudinal litorânea através do mapeamento sedimentológico das praias do município de Pontal do Paraná (PR). Monografia de Graduação. Bacharelado em Oceanografia, Setor de Ciências da Terra, Universidade Federal do Paraná, 66p.

Alves, E.S. \& Pezzuto, P.R. 2009. Effect of morphodynamics on annual average zonation pattern of benthic macrofauna of exposed sandy beaches in Santa Catarina, Brazil. Brazilian Journal of Oceanography, 57:189-203.

Blott, S.J. \& Pye, K. 2001. GRADISTAT: a grain size distribution and statistics package for the analysis of unconsolidated sediments. Earth Surface Processes and Landforms, 26:1237-1248.

Bons, P. \& Jessel, M.W. 1996. Image analysis of microstructures in natural and experimental samples. In: DE PAOR D.G. (ed), Structural Geology and Personal Computers. Pergamon, London, 135-166p.

Borzone, C.A.; Souza, J.R.B. \& Soares, A.G. 1996. Morphodynamic influence on the structure of inter and subtidal macrofaunal communities of subtropical sandy beaches. Revista Chilena de Historia Natural, 69:565-577.

Bouabid, R.; Nater, E.A. \& Barak, P. 1992. Measurement of pore size distribution in a lamellar Bt horizon using epifluorescence microscopy and image analysis. Geoderma, 53:309-328.

Defeo, O.; Jaramillo, E. \& Lyonnet, A. 1992. Community structure and intertidal zonation of the macroinfauna 
in the Atlantic coast of Uruguay. Journal of Coastal Research, 8:830-839.

Eshel, G.; Levy, G.J.; Mingelgrin, U. \& Singer, M.J. 2004. Critical evaluation of the use of laser diffraction for particle-size distribution analysis. Soil Science Society of America Journal 68:736-743.

Folk, R.L. \& Ward, W.C. 1957. Brazos River bar: a study in the significance of grain size parameters. Journal of Sedimentary Petrology, 27:3-26.

Fortey, N.J. 1995. Image analysis in mineralogy and petrology. Mineralogical Magazine, 59:177-178.

Francus, P. 1998. An image analysis technique to measure grain-size variation in thin sections of soft clastic sediments. Sedimentary Geology, 121:289-298.

Francus, P. \& Pirard, E. 2004. Testing for sources of errors in quantitative image analysis. In: FRANCUS P. (ed) Image analysis, sediments and paleoenvironments, Springer, Dordrecht, 87-102p.

Fritz, W.J. \& Moore, J.N. 1988. Basics of Physical Stratigraphy and Sedimentology. John Wiley and Sons Inc., New York, 371p.

Germán-Rodríguez, J. \& Uriarte, A. 2009. Laser Diffraction and Dry-Sieving Grain Size Analyses Undertaken on Fine- and Medium-Grained Sandy Marine Sediments: A Note. Journal of Coastal Research, 25(1):257-264.

Gibbs, R.J. 1972. The accuracy of particle-size analyses utilizing settling tubes. Journal of Sedimentary Petrology, 42:141-145.

Hardisty, J. 1990. Beaches: Form and Process. Unwin Hyman, London, 342p.

ISO 13320, 2009. Particle Size Analysis - Laser Diffraction Methods. 51p.

Kelly, R.N. \& Etzler, F.M. 2014. What is wrong with Laser diffraction? A critical review of current laser diffraction methods for Particle Size Analysis. Disponível em: http://www.donner-tech.com/ whats_wrong_with_ld.pdf. Acesso em 05 fevereiro 2015.

Krumbein, W.C. 1934. Size frequency distribution of sediments. Journal of Sedimentary Petrology, 4:65-77.

Krumbein, W.C. \& Pettijohn, F.J. 1938. Manual of Sediment Petrography. Appleton-Century Crofts Inc., New York, 549p.

Lira, C. \& Pina, P. 2011. Granulometry on classified images of sand grains. Journal of Coastal Research, 64(2):1697-1701.

Lira, C. \& Pina, P. 2009. Automated grain shape measurements applied to beach sands. Journal of Coastal Research, 56(2):1527-1531.

McLachlan, A. 1990. Dissipative beaches and macrofauna communities on exposed intertidal sands. Journal of Coastal Research, 6:57-71.
Otsu, N. 1979. A threshold selection method from graylevel histograms. IEEE Transactions on Systems, Man, and Cybernetics, 9(1):62-66.

Pina, P. \& Lira, C. 2009. Sediment image analysis as a method to obtain rapid and robust size measurements. Journal of Coastal Research, 56(2):1562-1566.

Rasband, W.S. 2013. ImageJ. Maryland, http://imagej. nih.gov/ij/.

Rubin, D.M. 2004. A simple autocorrelation algorithm for determining grain size from digital images of sediment. Journal of Sedimentary Research, 74:160-165.

Russ, J.C. 1990. Computer assisted microscopy, the measurement and analysis of images. Plenum Press, New York, 453p.

Sahu, B.K., 1965. Theory of sieving. Journal Sedimentary Petrology, 35:750-753.

Schindelin, J.; Arganda-Carreras, I.; Frise, E.; Kaynig, V.; Longair, M.; Pietzsch, T.; Preibisch, S.; Rueden, C.; Saalfeld, S.; Schmid, B.; Tinevez, J.Y.; White, D.J.; Hartenstein, V.; Eliceiri, K.; Tomancak, P. \& Cardona, A. 2012. Fiji: an open-source platform for biological-image analysis. Nature Methods, 9(7):676-682.

Schneider, C.A.; Rasband, W.S. \& Eliceiri, K.W. 2012. $\mathrm{NIH}$ Image to Image J: 25 years of image analysis. Nature Methods, 9:671-675.

Starkey, J. \& Samantaray, A.K. 1991. An evaluation of noise reduction filters, with particular reference to petrographic images. Journal of ComputerAssisted Microscopy, 3(4):171-188.

Starkey, J. \& Samantaray, A.K. 1994. A microcomputerbased system for quantitative petrographic analysis. Computers e Geosciences, 20(9):1285-1296.

Stojanović, Z.; Marković, S. \& Uskoković, D. 2012. Determination of Particle Size Distributions by Laser Diffraction. New Materials, 21:11-20.

Storti, F. \& Balsamo, F. 2010. Particle size distributions by laser diffraction: sensitivity of granular matter strength to analytical operating procedures. Solid Earth, 1:25-48.

Suguio, K. 1973. Introdução à sedimentologia. Edgard Blucher, São Paulo, 317p.

Syvitski, J.P.M.; Asprey, K.W. \& Clattenburg, D.A. 1991. Principles, design and calibration of settling tubes. In: SYVITSKI J.M.P. (ed.) Principles, Methods and Application of Particle Size Analysis. Cambridge University Press, Cambridge, 45-63p.

Wentworth, C.K. 1922. A scale of grade and class terms for clastic sediments. Journal of Geology, 30:377-392.

Submetido: Fevereiro/2015

Revisado: Setembro/2015 Aceito: Setembro/2015 